\title{
THE COVER ASSOCIATED TO A $(1,3)$-POLARIZED BIELLIPTIC ABELIAN SURFACE AND ITS BRANCH LOCUS
}

\author{
by GIANFRANCO CASNATI*
}

(Received 30th June 1997)

\begin{abstract}
Let $A$ be an abelian surface and let $|D|$ be a polarization of type $(1,3)$ on $A$. If $(A,|D|)$ is not a product of elliptic curves, such a polarization induces a finite morphism $\varrho: A \rightarrow \mathbb{P}_{\mathbf{C}}^{2}$ of degree 6 . In this paper we describe the branch locus of $\varrho$ when $A$ is bielliptic in the sense of $\mathrm{K}$. Hulek and S. H. Weintraub (see [13]), generalizing the results proved by Ch. Birkenhake and $H$. Lange in [4].
\end{abstract}

1991 Mathematics subject classification: $14 \mathrm{~K}$.

\section{Introduction}

Let $(A,|D|)$ be a $(1, d)$-polarized abelian surface. Here abelian surface means a surface $A$ with $\omega_{A \mid \mathbb{C}} \cong \mathcal{O}_{A}$ and $q(A)=2$ over $\mathbb{C}$ and $|D|$ is an ample linear system of type $(1, d)$ up to translation in $A$. It is known (see e.g. [14, Lemma 10.1.1]) that if

(O) $(A,|D|) \not \equiv\left(E_{1} \times E_{2}, p_{1}^{*} \mathcal{L}_{1} \otimes p_{2}^{*} \mathcal{L}_{2}\right)$ where $E_{i}$ is an elliptic curve, $p_{i}: E_{1} \times E_{2} \rightarrow E_{i}$ is the projection and $\mathcal{L}_{i} \in \operatorname{Pic}\left(E_{i}\right), i=1,2$,

then $|D|$ is free from base components. Therefore it induces a quasi-finite rational map $\varrho: A \rightarrow \mathbb{P}_{\mathrm{C}}^{d-1}$ such that $2 d=D^{2}=\operatorname{deg}(\varrho) \operatorname{deg}(\varrho(A))$. Since $C^{2} \geq 0$ for each irreducible curve $C$ on $A, \varrho$ is also finite.

There are many results about the behaviour of $\varrho$ with respect to $d$. If $d=2$ then $|D|$ has four base points and the map $\varrho$ has been studied in this case by W. Barth in [1]. If $d \geq 3$ then $|D|$ is base-point-free. When $d=4 \mathrm{C}$. Birkenhake, H. Lange, D. van Straten in [5] and F. Tovena in [22] have dealt with the morphism $\varrho$. Finally the case $d \geq 5$ has been described by $S$. Ramanan in [18].

In the case $d=3$ the map $\varrho$ is surjective and, since both $A$ and $\mathbb{P}_{C}^{2}$ are smooth, it is also flat (see [11, Exercise III 10.9]), i.e. a cover in the sense of [9]. In [4] a family $\mathcal{H}_{B L}$ of dimension 1 of such kind of surfaces is studied in details. In particular the branch locus $B_{\ell}$ of $\varrho: A \rightarrow \mathbb{P}_{\mathrm{C}}^{2}$ is described for each such $A$.

Really the main property of the surfaces $A$ corresponding to points in $\mathcal{H}_{B L}$ is the existence of a non-trivial involution $j_{A}: A \rightarrow A$. Hence they are bielliptic in the sense of

\footnotetext{
- Research supported by the framework of the AGE project, H.C.M. contract ERBCHRXCT 940557.
} 
[13]. Therefore there exists a double cover $\tau: A \rightarrow S$ onto a ruled surface $S$ with invariant $e(S)=-1$ over an elliptic curve $E$, and the cover $\varrho: A \rightarrow \mathbb{P}_{\mathbf{C}}^{2}$ factors as $\varrho=\sigma \circ \tau$ where $\sigma: S \rightarrow \mathbb{P}_{\mathrm{C}}^{2}$ is a cover of degree 3 .

The aim of this paper is to generalize to bielliptic abelian surfaces $A$ the mentioned results of [4] about the branch locus $B_{e}$ of the cover $\varrho:=\sigma \circ \tau: A \rightarrow \mathbb{P}_{\mathrm{c}}^{2}$ induced by $|D|$, using the theory of covers developed in $[15,21$ and 9$]$.

In Section 1 we study such a kind of cover $\sigma$, dealing with its branch locus and remification divisor. Moreover we describe the branch locus of the cover $\tau$. Finally we show how to recover the family $\mathcal{H}_{B L}$ as a particular case. Section 2 is devoted to the proof of the following theorem.

Theorem 0.1. There is a decomposition $B_{e}=2 C^{\prime}+C^{\prime \prime}$ into irreducible sextic curves birationally isomorphic to $E$.

The singularities of $C^{\prime}$ are nine cusps of type $A_{2}$. The singularities of $C^{\prime \prime}$ are nine points of type $A_{1}$ (possibly three by three infinitely near, i.e. three points of type $D_{4}$ ), lying on the cuspidal tangent lines at $C^{\prime}$.

In [9] a structure theorem for covers of degree $d$ between smooth varieties has been proved. Such a result has been used in order to give a complete characterization of covers of low degree $d$, namely $3 \leq d \leq 5$ (see $[9,7,8]$ ).

More precisely if $\varrho: X \rightarrow Y$ is a cover of degree $d \geq 3$ and both $X$ and $Y$ are smooth, Theorem 2.1 of [9] asserts the existence of a locally free $\mathcal{O}_{Y}$-sheaf $\mathcal{E}$ of rank $d-1$, natural splittings

$$
\begin{aligned}
& \varrho_{*} \omega_{X \mid Y} \cong \mathcal{O}_{Y} \oplus \mathcal{E}, \\
& \varrho_{*} \mathcal{O}_{X} \cong \mathcal{O}_{Y} \oplus \check{\mathcal{E}},
\end{aligned}
$$

and an embedding $i: X \hookrightarrow \mathbb{P}:=\mathbb{P}(\mathcal{E})$ such that $\varrho=\pi \circ i(\pi: \mathbb{P} \rightarrow Y$ is the projection) and

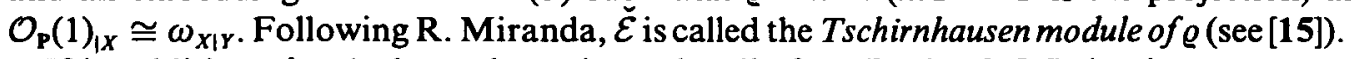

If in addition, $d \geq 4$, there also exists a locally free $\mathcal{O}_{Y}$-sheaf $\mathcal{F}$ fitting into a sequence of the form

$$
0 \longrightarrow \mathcal{F} \stackrel{\eta}{\longrightarrow} \mathcal{S}^{2} \mathcal{E} \stackrel{\varphi}{\longrightarrow} \varrho_{*} \omega_{X \mid Y}^{2} \longrightarrow 0 .
$$

Notice that $\mathcal{F}$ has rank $N_{d}:=\frac{d(d-3)}{2}$.

If $d \geq 4$ (resp. $d=3$ ), via $\Phi_{d}: H^{0}\left(Y, \check{\mathcal{F}} \otimes \mathcal{S}^{2} \mathcal{E}\right) \stackrel{\sim}{\rightarrow} H^{0}\left(\mathbb{P}, \pi^{*} \check{\mathcal{F}}(2)\right)$ (resp. $\Phi_{3}: H^{0}\left(Y, \mathcal{S}^{3} \mathcal{E} \otimes\right.$ $\left.\left.\operatorname{det} \mathcal{E}^{-1}\right) \stackrel{\sim}{\rightarrow} H^{0}\left(\mathbb{P}, \mathcal{O}_{\mathbf{P}}(3) \otimes \pi^{*} \operatorname{det} \mathcal{E}^{-1}\right)\right)$ we obtain a morphism $\delta:=\Phi_{d}(\eta): \pi^{*} \mathcal{F}(-2) \rightarrow \mathcal{O}_{\mathbf{P}}$ (resp. $\delta:=\Phi_{3}(\eta): \mathcal{O}_{\mathrm{P}}(-3) \otimes \pi^{*} \operatorname{det} \mathcal{E} \rightarrow \mathcal{O}_{\mathrm{p}}$ ) and $X=D_{0}(\delta) \subseteq \mathbb{P}$. If $d \geq 5$ such a section $\eta$ cannot be general since $\operatorname{codim}_{\mathrm{P}}(X)=d-2<N_{d}$. If $d=5$, it can be proved (see [7] for the details) that such $\eta$ 's belong to the image of a natural quadratic map

$$
H^{0}\left(Y, \Lambda^{2} \mathcal{F} \otimes \mathcal{E} \otimes \operatorname{det} \mathcal{E}^{-1}\right) \rightarrow H^{0}\left(Y, \check{\mathcal{F}} \otimes \mathcal{S}^{2} \mathcal{E}\right) .
$$

Unfortunately for $d \geq 6$ there is not such a satisfactory theory. 
Anyhow, given an arbitrary $(1,3)$-polarized abelian surface $(A,|D|)$, it is interesting to compute the sheaves $\mathcal{E}$ and $\mathcal{F}$ of the associated cover $\varrho: A \rightarrow \mathbb{P}_{\mathrm{c}}^{2}$. In Section 3 we prove the following theorem.

Theorem 0.4. Let $(A,|D|)$ be a $(1,3)$-polarized abelian surface satisfying $(\mathcal{O})$. Then the sheaves $\mathcal{E}$ and $\mathcal{F}$ corresponding to the cover $\varrho: A \rightarrow \mathbb{P}_{\mathrm{C}}^{2}$ are

$$
\begin{aligned}
\mathcal{E} & \cong \mathcal{O}_{\mathbf{P}_{\mathbf{C}}^{2}}(3) \oplus \Omega_{\mathbf{P}_{\mathbf{C}}^{2} \mid \mathrm{C}}^{1}(3)^{\oplus 2}, \\
\mathcal{F} & \cong \mathcal{S}^{2} \Omega_{\mathbf{P}_{\mathbf{C}}^{2} \mid \mathrm{C}}^{1}(6)^{\oplus 3} .
\end{aligned}
$$

Finally, in Section 4, we will give a complete description of the structure of the map $\varrho$ for bielliptic surfaces.

Theorem 0.5. Let $\varrho: A \rightarrow \mathbb{P}_{\mathrm{C}}^{2} a(1,3)$-polarized abelian surface and let $i: A \hookrightarrow \mathbb{P}$ the embedding above. Then $A$ is bielliptic if and only if the restriction to $A$ of the projection $\bar{\pi}: \mathbb{P}\left(\mathcal{O}_{\mathrm{P}_{\mathrm{C}}^{2}}(3) \oplus \Omega_{\mathrm{P}_{\mathrm{C}}^{2} \mid \mathrm{C}}^{1}(3) \oplus \Omega_{\mathrm{P}_{\mathrm{C}}^{2} \mid \mathrm{C}}^{1}(3)\right) \rightarrow \mathbb{P}\left(\Omega_{\mathrm{P}_{\mathrm{C}} \mid \mathrm{C}}^{1}(3)\right)$ from $\mathbb{P}\left(\mathcal{O}_{\mathrm{P}_{\mathrm{C}}^{2}}(3) \oplus \Omega_{\mathrm{P}_{\mathrm{C}}^{2} \mid \mathrm{C}}^{1}(3)\right)$ induced by the decomposition 0.4 .1 is a morphism whose image is smooth.

For all the notations and definitions used in the paper we refer to [11].

\section{1. (1,3)-Polarized bielliptic abelian surfaces}

Let $E$ be an elliptic curve and consider the unique ruled surface $S$ over $E$ with invariant $e(S)=-1$. Then there exists an indecomposable locally free $\mathcal{O}_{E}$-sheaf $\mathcal{H}$ of rank 2, fitting into the sequence

$$
0 \rightarrow \mathcal{O}_{E} \rightarrow \mathcal{H} \rightarrow \mathcal{O}_{E}(P) \rightarrow 0
$$

such that $S \cong \mathbb{P}(\mathcal{H}) \stackrel{e}{\longrightarrow} E$. Fixing such an isomorphism, let $\left\{C_{0}\right\}=\left|\mathcal{O}_{s}(1)\right|$. Notice that $C_{0}^{2}=1$ and $\operatorname{Pic}(S) \cong \mathbb{Z} C_{0} \oplus e^{*} \operatorname{Pic}(E)$.

Proposition 1.1. If $Q \in E$, then $\left|C_{0}+e^{*} Q\right|$ induces a cover $\sigma: S \rightarrow \mathbb{P}_{\mathrm{C}}^{2}$ of degree 3 with Tschirnhausen module $\Omega_{\mathrm{P}_{\mathrm{C}}^{2} \mathrm{I}}^{1}$

Proof. If $D \in\left|C_{0}+Q f\right|$ then $D^{2}=3$ and $\operatorname{dim}\left|C_{0}+e^{*} Q\right|=2$. Moreover $\left|C_{0}+e^{*} Q\right|$ is ample and base-point-free (see [10, Proposition 3.4 and 3.5]).

If $r \in \breve{\mathbb{P}}_{\mathrm{C}}^{2}$ and $C:=\sigma^{-1}(r)$ is smooth then the branch locus of $\sigma_{\mid C}$ has degree 6 by the theorem of Hurwitz. Thus the branch locus $B_{a}$ of $\sigma$ has degree 6 , hence $c_{1}(\mathcal{E})=-3$ for the Tschirnhausen module $\mathcal{E}$ of $\sigma$. On the other hand computing $\chi\left(\mathcal{O}_{s}\right)$ for the triple cover $\sigma$ (see [17, Section 8 or 15 , Section 10]) one obtains $c_{2}(\check{\mathcal{E}})=3$. Normalizing $\check{\mathcal{E}}$ we then get $c_{1}\left(\check{\mathcal{E}}_{\text {norm }}\right)=-1, c_{2}\left(\check{\mathcal{E}}_{\text {norm }}\right)=1$. On the other hand

$$
3=h^{0}(E, \mathcal{H}(Q))=h^{0}\left(S, \sigma^{*} \mathcal{O}_{\mathbb{P}_{\mathrm{C}}^{2}}(1)\right)=h^{0}\left(\mathbb{P}_{\mathrm{C}}^{2}, \mathcal{O}_{\mathrm{P}_{\mathrm{C}}^{2}}(1)\right)+h^{0}\left(\mathbb{P}_{\mathrm{C}}^{2}, \check{\mathcal{E}}_{\text {norm }}\right)
$$


therefore $\check{\mathcal{E}}_{\text {norm }}$ is stable. We then conclude that $\check{\mathcal{E}}_{\text {norm }} \cong \Omega_{\text {Pé }_{\mathrm{C}}^{1} \text { |C }}^{1}(1)$ (see $[16$, p. 246]).

Proposition 1.2. The set $H$ of sections of $H^{0}\left(\mathbb{P}_{\mathrm{C}}^{2}, \mathcal{S}^{3} \Omega_{\mathrm{P}_{\mathrm{C}}^{2} \mathrm{C}}^{1}(6)\right)$ inducing covers (as explained in the introduction) $\sigma: S \rightarrow \mathbb{P}_{\mathrm{C}}^{2}$ of degree 3 with a smooth $S$ is open and dense.

Each such cover $\sigma$ is non-cyclic and there is an elliptic curve $E$, and a point $Q \in E$ such that $S$ is the unique ruled surface over $E$ with invariant $e(S)=-1$ and the pull back to $S$ of the linear system of lines is $\left|C_{0}+e^{*} Q\right|$.

Proof. The first statement follows from [9, Theorem 3.6], since $\mathcal{E} \cong \Omega_{\mathrm{p}_{\mathrm{C}}^{2} \mid \mathrm{c}}^{1}(3)$. Moreover $\sigma$ is not cyclic since its Tschirnhausen module does not split.

Notice that $\chi\left(\mathcal{O}_{s}\right)=K_{s}^{2}=0$ (see [17, Section 8] or [15, Section 10]) hence $S$ is minimal. Thus $S$ is either a ruled surface $\mathbb{P}(\mathcal{H})$ over an elliptic curve $E$ or a surface with Kodaira dimension $\kappa(D) \geq 0$.

Let $D \in\left|\sigma^{*} \mathcal{O}_{P_{c}^{2}}(1)\right|$. In the second case one would have $K_{S} \cdot D \geq 0$. On the other hand, using projection formula and the isomorphism $\sigma_{*} \omega_{S \mid \mathrm{C}}^{2} \cong \mathcal{S}^{2} \Omega_{\mathrm{PC}_{\mathrm{C}}^{2} \mathrm{C}}^{1}$ (see [17, Section 8 or 9, formula 5.1.2]), one has

$$
2 K_{s} \cdot D=\chi\left(\mathcal{O}_{s}\right)-\chi\left(\omega_{S \mid C}^{2}\right)-\chi\left(\mathcal{O}_{s}(D)\right)+\chi\left(\omega_{s \mid C}^{2}(D)\right)=-6 .
$$

Thus $S \cong \mathbb{P}(\mathcal{H})$ for some locally free $\mathcal{O}_{E}$-sheaf $\mathcal{H}$ of rank 2 on an elliptic curve $E$. Let $C_{0} \subseteq S$ be a section of minimal self-intersection $C_{0}^{2}=-e(S)$. Then $D \in\left|a C_{0}+e^{*} b\right|$ where $a \geq 1$ and $\mathfrak{b}$ is a divisor on $E$. Moreover

$$
3=a^{2} C_{0}^{2}+2 a b
$$

where $b=\operatorname{deg}(\mathfrak{b})$. It follows that $e(S)$ is odd. If $e(S)>0$, since $D$ is ample then $b+a C_{0}^{2}>0$ ([10, Proposition 3.4]), hence $3+a^{2} C_{0}^{2}>0$. It follows $C_{0}^{2}=-1, a=1$ and $b=2$, which is absurd since then $D$ would not be free from base points.

If $e(S)=-1$ then $\mathcal{H}$ fits into

$$
0 \rightarrow \mathcal{O}_{E} \rightarrow \mathcal{H} \rightarrow \mathcal{O}_{E}(P) \rightarrow 0 .
$$

Since $2 b=3 / a-a^{2} C_{0}^{2}$ then $a=1,3$. If $a=3$ then $2 K_{s} \cdot D=-6-4 b=-6$ if and only if $b=0$, contradicting formula 1.2.2 again. We conclude that $a=1$ and $b=Q \in E$.

Now we deal with the branch locus $B_{\sigma}$ and the ramification divisor $R_{\sigma}$ of the cover $\sigma: S \rightarrow \mathbb{P}_{\mathrm{C}}^{2}$ corresponding to a section $\vartheta \in H^{0}\left(\mathbb{P}_{\mathrm{C}}^{2}, \mathcal{S}^{3} \Omega_{\mathbf{P}_{\mathrm{C}}^{2} \mid \mathrm{C}}^{1}(6)\right)$. We always refer to the careful description of the branch locus of a triple cover due to R. Miranda (see [15]).

More precisely $B_{\sigma}$ is singular at $x \in \mathbb{P}_{C}^{2}$ if and only if $\sigma$ is totally ramified over $x$ ([15, Lemma 4.8]). If $x \in \operatorname{Sing}\left(B_{\sigma}\right)$ then it is a double point with one tangent, and if it is also an isolated singularity, then $x$ is a point of type $A_{3 k-1}$ for some $k \geq 1$ (see [15, Corollary 5.8 and its proof]).

Lemma 1.3. Assume that $B_{\sigma}$ is reduced. Then its singularities are $a_{2}$ points of type $A_{2}$ and $a_{5}$ points of type $A_{5}$. Moreover $2 a_{5}+a_{2}=9$. 
Proof. The first part of the statement follows from [15, Corollary 5.8], its proof and the fact that $\operatorname{deg}\left(B_{\sigma}\right)=6$.

Let $\vartheta \in H^{0}\left(\mathbb{P}_{\mathbf{C}}^{2}, S^{3} \Omega_{\mathbf{P}_{\mid}^{2} \mathbf{C}}^{1}(6)\right)$ correspond to $\sigma: S \rightarrow \mathbb{P}_{\mathbf{C}}^{2}$. As shown in Proposition 3.9 and Lemma 10.1 of [15] there is a natural map

$$
\alpha: H^{0}\left(\mathbb{P}_{\mathbf{C}}^{2}, \mathcal{S}^{3} \Omega_{\mathbf{P}_{\mathbf{C}}^{2} \mid \mathrm{C}}^{1}(6)\right) \rightarrow \operatorname{Hom}_{\mathcal{O}_{\mathbf{P}_{\mathbf{C}}^{2}}}\left(\Omega_{\mathbf{P}_{\mathbf{C}}^{2} \mid \mathrm{C}}^{1}(-3), \mathcal{S}^{2} \Omega_{\mathbf{P}_{\mathbf{C}}^{2} \mid \mathrm{C}}^{1}\right)
$$

inducing as an exact sequence

$$
0 \rightarrow \Omega_{\mathbf{P}_{\mathbf{C}}^{2} \mid \mathbf{C}}^{1}(-3) \stackrel{\alpha(s)}{\rightarrow} \mathcal{S}^{2} \Omega_{\mathbf{P}_{\mathbf{C}}^{2} \mid \mathbf{C}}^{1} \rightarrow \mathcal{O}_{\mathbf{P}_{\mathbf{C}}^{2}} \rightarrow \mathcal{O}_{T} \rightarrow 0,
$$

where the support of $T$ is the set of points of total ramification of $\sigma$. A Chern class computation shows that $\operatorname{deg}(T)=9$.

Locally at $x \in T$

$$
\mathfrak{J}_{T, x}=\left(a^{2}-b d, a d-b c, d^{2}-a c\right) \mathcal{O}_{\mathrm{P}_{C}^{2}, x}
$$

where $a, b, c, d \in \mathcal{O}_{\mathrm{P}_{\mathrm{c}}^{2}, x}$ are the local functions around $x$ defining the cover $\sigma$ (see Sections 3 and 4 of [15]). Let $\mathfrak{M}$ be the maximal ideal of $\mathcal{O}_{\mathbf{P}_{\mathbf{c}}^{3}, x}$.

If $b, c \in \mathfrak{M}$ then also $a, d \in \mathfrak{M}$, thus the fibre of $\sigma$ over $x$ would be isomorphic to

$$
\mathbb{C}[z, w] /\left(z^{2}, z w, w^{2}\right)
$$

which is not Gorenstein, hence $S$ could not be smooth over $x$.

Let $b \notin \mathfrak{M}$. Locally the equation of $S$ is $z^{3}+g z+h=0$, where $h:=3 a b d-2 a^{3}-b^{2} c$, $g:=3\left(b d-a^{2}\right)$ (see [15, Remark 2.8.1]). In particular $\mathfrak{J}_{T, x}=(g, h) \mathcal{O}_{\mathbf{P}_{c, x}^{2}}$ and the local equation of $B_{a}$ around $x$ is $27 h^{2}+4 g^{3}=0$. Since $h \in \mathfrak{M} \backslash \mathfrak{M}^{2}$ (see [15, Lemma 5.7]), thus $\boldsymbol{x}$ is of type $A_{2}$ (resp. $A_{5}$ ) if and only if $g \in \mathfrak{M} \backslash \mathfrak{M}^{2}$ (resp. $g \in \mathfrak{D}^{2}$ ) at $x$, that is if and only if $x$ has degree 1 (resp. 2) inside $T$.

Theorem 1.4. $B_{\sigma}$ is an irreducible sextic curve birationally isomorphic to the curve E. Its singularities are nine points of type $A_{2}$.

Proof. Notice that each irreducible component $C \subseteq R_{\sigma}$ is mapped birationally onto $\sigma(C)$, since $\operatorname{deg}(\sigma)=3$.

Since $R_{\sigma} \cdot\left(C_{0}+e^{*} Q\right)=6$ then $\operatorname{deg}\left(B_{\sigma}\right)=6$. Assume that $B_{\sigma}$ is reducible. Then it must be reduced, otherwise it would have at least a triple point, a contradiction by Lemma 4.8 and Corollary 5.8 of [15]. It follows that $R_{\sigma} \in\left|C_{0}+e^{*}(P+3 Q)\right|$ is reducible too, hence $R_{\sigma}$ contains a fibre $e^{*} S$.

Since $e^{*} S \cdot\left(C_{0}+e^{*} Q\right)=1$ then $e^{*} S$ is mapped on a line $r \subseteq B_{\sigma}$. If $B_{\sigma}$ contains another line $r^{\prime}$ then $r \neq r^{\prime}$ hence $B_{\sigma}$ has a node, an absurd. It follows that the residual divisor $B:=B_{\sigma}-r$ must be irreducible.

The points of $B \cap r$ are simple on $B$ and they are images of points of total ramification of $\sigma$. Since $e^{*} S \cdot\left(R_{\sigma}-e^{*} S\right)=1$ it follows that $r \cap B$ is exactly one point $x$. Such a point is necessarily a flex on $B$ and $r$ is its inflectional tangent. 
The other singularities of $B_{\sigma}$ are also singularities of $B$, thus they must be points of type $A_{2}$. Since $B$ is birationally isomorphic to $E$ then $B_{\sigma}$ must have exactly $a_{2}=5$ points of type $A_{2}$ and $a_{5}=1$ point of type $A_{5}$ (namely $x$ ). We conclude that in this case $2 a_{5}+a_{2}=7 \neq 9$.

Hence we have proved that $B_{\sigma}$ is irreducible. If it is not reduced, then $\sigma$ would be totally ramified, whence cyclic ([21, Proposition 3.1]).

We conclude that $B_{\sigma}$ is irreducible and reduced, thus it is birationally equivalent to $E$. In particular the formula of Clebsch yields $1=p_{g}\left(B_{\sigma}\right)=10-3 a_{5}-a_{2}$, thus $a_{2}+3 a_{5}=a_{2}+2 a_{5}=9$ which implies $a_{5}=0$.

In Proposition 3.2 of [10], it is proved that the linear system $\left|\omega_{s \mid C}^{-2}\right|=\left|4 C_{0}-2 e^{*} P\right|$ has dimension 1 and its generic member is a smooth irreducible curve. We now produce the two-dimensional family of bielliptic abelian surfaces with a $(1,3)$ polarization.

Proposition 1.5. Let $\sigma: S \rightarrow \mathbb{P}_{\mathrm{C}}^{2}$ be as in Proposition 1.2 and let $\tau: A \rightarrow S$ be the double cover branched along a general divisor $B_{\tau} \in\left|\omega_{S \mid C}^{-2}\right|$. Then $\left(A,\left|\tau^{*}\left(C_{0}+e^{*} Q\right)\right|\right)$ is a $(1,3)$ polarized bielliptic abelian surface.

Conversely each $(1,3)$-polarized bielliptic abelian surface $(A,|D|)$ arises in this way.

Proof. The general element of $\left|\omega_{s \mid c}^{-2}\right|$ is smooth and irreducible, thus $A$ is smooth. Moreover $q(A)=2, p_{g}(A)=1$ and $\omega_{A \mid \mathrm{C}} \cong \mathcal{O}_{A}$ (see [2, Lemma 17.1 of Chapter I]), thus $A$ is abelian.

The map $\varrho:=\sigma \circ \tau: A \rightarrow \mathbb{P}_{\mathrm{C}}^{2}$ is a cover of degree 6, then $\left|\tau^{*}\left(C_{0}+e^{*} Q\right)\right|=\left|\varrho^{*} \mathcal{O}_{\mathbb{P}_{\mathrm{C}}^{2}}(1)\right|$ is ample, hence it must be a $(1,3)$-polarization. The map $\tau$ induces a non-trivial involution $j_{A}: A \rightarrow A$, thus $A$ is bielliptic.

The converse follows trivially from (i) and (ii) of Proposition 4.4 in [13].

Remark 1.6. Since $\left(4 C_{0}-2 e^{*} Q\right)^{2}=0$ then we get a fibration $\varphi: S \rightarrow \mathbb{P}_{\mathrm{c}}^{1}$, whose generic fibre is a smooth elliptic curve.

Moreover $\chi\left(\mathcal{O}_{s}\right)=0$ hence $\varphi$ is isotrivial, its singular fibres are multiple of smooth curves (see Lemma 1.1 of [20]), and $\varphi$ has exactly three double fibres by Proposition 3.2 of $[10]$.

Consider the residual divisor $R_{0}:=\sigma^{*} B_{\sigma}-2 R_{\sigma} \in\left|4 C_{0}-2 e^{*} P\right|$. Then the restriction of $\sigma$ to $R_{0} \backslash \sigma^{-1}$ (Sing $\left(B_{\sigma}\right)$ ) is an isomorphism onto $B_{\sigma} \backslash \operatorname{Sing}\left(B_{\sigma}\right)$. Moreover Lemma 5.9 of [15] asserts the smoothness of $R_{0}$ also at the points of total ramification. We conclude that $R_{0}$ is globally smooth, hence irreducible, and $\sigma_{\mid R_{0}}$ is birational onto $B_{0}$. It follows that $R_{0} \cong E$ and that all the smooth fibres of $\varphi$ are isomorphic to $E$.

The fibres of the map $\varphi \circ \tau: A \rightarrow \mathbb{P}_{\mathrm{C}}^{1}$ are double étale covers of the curves in $\left|4 C_{0}-2 e^{*} P\right|$, since $B_{\tau} \cdot\left(4 C_{0}-2 e^{*} P\right)=0$. It follows that they are not connected (see [3, Exercise IX 1]), hence the Stein factorization of $\varphi \circ \tau$ gives rise to a commutative diagram 


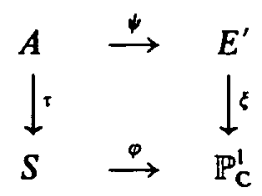

where $E^{\prime}$ is a smooth elliptic curve and $\xi$ is a double cover.

The map $\varphi_{\mid C_{0}}: C_{0} \rightarrow \mathbb{P}_{\mathrm{C}}^{1}$ is a double cover since $C_{0} \cdot\left(4 C_{0}-2 e^{*} P\right)=2$. Its branch points are exactly the critical values of $\varphi \circ \tau$. Therefore they coincide with the branch points of $\xi$. In particular $E^{\prime} \cong C_{0} \cong E$, and we have an exact sequence of abelian varieties

$$
0 \longrightarrow E \longrightarrow A \stackrel{\psi}{\longrightarrow} E \longrightarrow 0
$$

(see also [3, Example IX 4.3]).

Notice that $A \cong(E \times F) / G$ where $G:=\mathbb{Z}_{2} \times \mathbb{Z}_{2}$ (see [13, Proposition 4.1]) and $F / G \cong E$ (see [20, Theorem 1.2]).

In the following example, choosing $R_{0}=B_{\tau}$, we obtain the family studied in [4].

Example 1.7. Let $\sigma: S \rightarrow \mathbb{P}_{\mathrm{c}}^{2}$ be as above. Since the Tschirnhausen module of $\sigma$ does not split, then $\sigma$ is not cyclic. According to [21] we can build the discriminant $D\left(S \mid \mathbb{P}_{\mathbf{C}}^{2}\right)$ of $\sigma$ and we have a commutative square

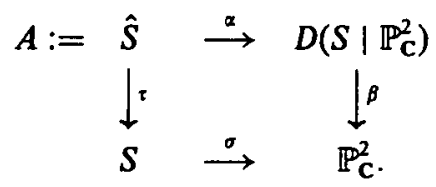

Theorem 1.4 above and [21, Proposition 3.4] give us the following results:

(i) $\beta$ is a double cover branched along $B_{\sigma}$ and $D\left(S \mid \mathbb{P}_{\mathrm{C}}^{2}\right)$ is normal with 9 singular points of type $A_{2}$;

(ii) $\alpha$ is a cyclic triple cover of $D\left(S \mid \mathbb{P}_{\mathrm{C}}^{2}\right)$ branched only at $\operatorname{Sing}\left(D\left(S \mid \mathbb{P}_{\mathrm{C}}^{2}\right)\right)$ and $A:=\hat{S}$ is smooth;

(iii) $S$ is the quotient of $A:=\hat{S}$ via an involution.

In particular $D\left(S \mid \mathbb{P}_{\mathrm{C}}^{2}\right)$ is a singular $K$ 3-surface and $A$ is a bielliptic abelian surface. Again $\varrho:=\sigma \circ \tau$ is a cover of degree 6 and $\varrho^{*} \mathcal{O}_{\mathrm{P}_{\mathrm{C}}^{2}}(1)$ is a polarization of type $(1,3)$ on A.

Notice that Lemma 1.4 of [21] implies that the reduced branch locus of $\varrho$ is $B_{\sigma}$, thus the branch locus of $\varrho$ satisfies $B_{e}=3 B_{\sigma}$. It follows that $B_{\imath}=R_{0}$. Moreover $\varrho$ is a Galois cover with Galois group $G_{3}$ (see again [21]). 


\section{The equation of the branch locus $B_{e}$}

In this section we will describe the branch locus $B_{Q}$ of the cover $\varrho: A \rightarrow \mathbb{P}_{\mathrm{C}}^{2}$, when $A$ is bielliptic. To this purpose we denote by $t_{\alpha}: A \rightarrow A$ the translation by $a \in A$ and we set $|D|:=\left|\varrho^{*} \mathcal{O}_{\mathbf{P}_{\mathrm{C}}^{2}}(1)\right|$.

Since $D$ is a polarization of type $(1,3)$, the morphism $\varrho: A \rightarrow \mathbb{P}_{\mathbf{C}}^{2}$ is invariant with respect to the group

$$
K(D):=\left\{a \in A\left|t_{\alpha}^{*} D \in\right| D \mid\right\} \cong \mathbb{Z}_{3} \times \mathbb{Z}_{3} \subseteq P G L_{3} .
$$

Proposition 2.1. There exists a decomposition into $K(D)$-invariant sextic curves $B_{e}=2 B_{\sigma}+C_{e}$.

Proof. Obviously $B_{\ell}=2 B_{\sigma}+\sigma_{*} B_{\tau}$. Let $C_{\ell}:=\sigma_{*} B_{\tau}$ and fix a general line $\ell \in \mathbb{P}_{\mathrm{C}}^{2}$. Then $C:=\varrho^{-1}(\ell) \in|D|$ is a smooth irreducible curve of genus 4 , by adjunction formula. Hence the theorem of Hurwitz applied to $C$ yields $\operatorname{deg}\left(B_{Q}\right)=18$, whence $\operatorname{deg}\left(C_{Q}\right)=6$.

Since $B_{e}$ is $K(D)$-invariant, thus the two curves $C_{e}$ and $B_{\sigma}$ must be invariant too.

With a suitable choice of the coordinates $x_{0}, x_{1}, x_{2}$ in $\mathbb{P}_{\mathrm{C}}^{2}$, we can assume that $K(D) \subseteq P G L_{3}$ is generated by the classes of

$$
\left(\begin{array}{lll}
0 & 1 & 0 \\
0 & 0 & 1 \\
1 & 0 & 0
\end{array}\right), \quad\left(\begin{array}{lll}
1 & 0 & 0 \\
0 & \zeta & 0 \\
0 & 0 & \zeta^{2}
\end{array}\right)
$$

where $\zeta \neq 1, \zeta^{3}=1$. The $K(D)$-orbit $O(x)$ of a point $x \in \mathbb{P}_{\mathbf{c}}^{2}$ contains at most nine distinct points. If $O(x)$ contains less than nine points then it coincides with one of the following:

$$
\begin{array}{ll}
O_{0}:=\{[1,0,0],[0,1,0],[0,0,1]\}, & O_{1}:=\left\{[1,1,1],\left[1, \zeta, \zeta^{2}\right],\left[1, \zeta^{2}, \zeta\right]\right\} \\
O_{2}:=\{[1,1, \zeta],[1, \zeta, 1],[\zeta, 1,1]\}, & O_{3}:=\left\{\left[1,1, \zeta^{2}\right],\left[1, \zeta^{2}, 1\right],\left[\zeta^{2}, 1,1\right]\right\}
\end{array}
$$
form

A simple computation shows that each $K(D)$-invariant sextic has an equation of the

$$
\begin{aligned}
f\left(x_{0}, x_{1}, x_{2}\right):= & a\left(x_{0}^{6}+x_{1}^{6}+x_{2}^{6}\right)+b\left(x_{0}^{3} x_{1}^{3}+x_{0}^{3} x_{2}^{3}+x_{1}^{3} x_{2}^{3}\right)+ \\
& +c x_{0} x_{1} x_{2}\left(x_{0}^{3}+x_{1}^{3}+x_{2}^{3}\right)+d x_{0}^{2} x_{1}^{2} x_{2}^{2}=0,
\end{aligned}
$$

for some $[a, b, c, d] \in \mathbb{P}_{\mathrm{C}}^{3}$. Let $C \subseteq \mathbb{P}_{\mathrm{C}}^{2}$ be the corresponding curve. We have a rational map

$$
\varphi_{f}:=\left(\frac{\partial f}{\partial x_{0}}, \frac{\partial f}{\partial x_{1}}, \frac{\partial f}{\partial x_{2}}\right): \mathbb{P}_{c}^{2} \rightarrow \mathbb{P}_{c}^{\check{2}}
$$


and, computing $\partial f / \partial x_{i}$, one easily checks that $\varphi_{f}$ is $K(D)$-equivalent. It follows that the dual curve $\check{C} \subseteq \mathbb{P}_{\mathrm{C}}^{2}$ is also $K(D)$-invariant. Let $g$ be its equation in ${\widetilde{\mathbb{P}_{\mathrm{C}}^{2}}}^{2}$ with coordinates $y_{0}, y_{1}, y_{2}$. We have

$$
\psi_{\theta}:=\left(\frac{\partial g}{\partial x_{0}}, \frac{\partial g}{\partial x_{1}}, \frac{\partial g}{\partial x_{2}}\right): \stackrel{\mathbb{P}_{\mathrm{C}}^{2}}{\rightarrow} \rightarrow \mathbb{P}_{\mathrm{C}}^{2},
$$

and it is well known that the biduality $\psi_{g} \circ \varphi_{f}$ is the identity on $C$ (in particular $C$ and $\check{C}$ are birational).

Since $B_{\sigma}$ has nine points of type $A_{2}$, the ordinary formula of Plücker implies that $\breve{B}_{\sigma}$ is a smooth cubic. Therefore its equation is

$$
y_{0}^{3}+y_{1}^{3}+y_{2}^{3}-3 \lambda y_{0} y_{1} y_{2}=0, \quad \lambda^{3} \neq 1 .
$$

Taking into account Section 1 of [4], by biduality we get that the equation of $B_{\sigma}$ with respect to the above system of coordinates is

$$
\begin{aligned}
f_{2}\left(x_{0}, x_{1}, x_{2}\right):= & \left(x_{0}^{6}+x_{1}^{6}+x_{2}^{6}\right)+2\left(2 \lambda^{3}-1\right)\left(x_{0}^{3} x_{1}^{3}+x_{0}^{3} x_{2}^{3}+x_{1}^{3} x_{2}^{3}\right) \\
& -6 \lambda^{2} x_{0} x_{1} x_{2}\left(x_{0}^{3}+x_{1}^{3}+x_{2}^{3}\right)-3 \lambda\left(\lambda^{3}-4\right) x_{0}^{2} x_{1}^{2} x_{2}^{2}=0,
\end{aligned}
$$

where $\lambda^{3} \neq 1$. Notice that $O_{i} \cap B_{\sigma}=\emptyset$ and that $\operatorname{Sing}\left(B_{\sigma}\right)=O([\lambda, 1,1])$.

Remark 2.4. Let $\gamma, \delta \in P G L_{3}$ be classes of the matrices

$$
\left(\begin{array}{ccc}
1 & 0 & 0 \\
0 & \zeta^{2} & 0 \\
0 & 0 & \zeta^{2}
\end{array}\right), \quad\left(\begin{array}{ccc}
1 & 1 & 1 \\
1 & \zeta^{2} & \zeta \\
1 & \zeta & \zeta^{2}
\end{array}\right)
$$

The group $G:=\langle\gamma, \delta\rangle$ is well-known to be isomorphic to the alternating group $\mathcal{A}_{4}$ of order 4 ([6, Section 7.3]). The elements of order two of $G$ form a normal subgroup $G_{0} \cong \mathbb{Z}_{2} \times \mathbb{Z}_{2} \unlhd G, \gamma$ generates a cyclic subgroup $G_{1} \cong \mathbb{Z}_{3}$ and $G \cong G_{0} \rtimes G_{1}$.

The polynomials $f_{\lambda}$ and $f_{\mu}$ represent birationally isomorphic curves if and only if they lie in the same $G$-orbit, i.e. if and only if there is $g \in G$ such that $g\left(f_{k}\right)=f_{\mu}$ (see [4, Section 1] and [6]). The group $G$ induces an action on $\mathbb{C} \backslash\left\{1, \zeta, \zeta^{2}\right\}$ given by

$$
\gamma(\lambda):=\zeta \lambda, \quad \delta(\lambda):=\frac{\lambda+2}{\lambda-1} .
$$

With respect to this action $g\left(f_{\lambda}\right)=f_{g(\lambda)}, g \in G$. Then $f_{\lambda}$ and $f_{\mu}$ represent birationally isomorphic curves if and only if $g(\lambda)=\mu$. For such a pair $(\lambda, \mu)$, some easy computation shows the existence of $g \in G_{0}$, depending on $(\lambda, \mu)$, and sending $(\lambda, \mu)$ to $\left(\lambda^{\prime}, \mu^{\prime}\right)$ where $\mu^{\prime} \in\left\{\lambda^{\prime}, \gamma\left(\lambda^{\prime}\right), \gamma^{2}\left(\lambda^{\prime}\right), \delta\left(\lambda^{\prime}\right)\right\}$.

Remark 2.5. We claim that if $y \in B_{\mathfrak{\tau}} \cap R_{\sigma}$ and $x:=\sigma(y) \notin \operatorname{Sing}\left(B_{\sigma}\right)$, then the tangent space of $C_{Q}$ at $x$ contains the tangent space of $B_{\sigma}$ at $x$. 
Since the assertion is local we can consider $\sigma: \operatorname{spec}(B) \rightarrow \operatorname{spec}(A) \subseteq \mathbb{P}_{\mathrm{C}}^{2}$, where $A$ is a ring with maximal ideal $\mathfrak{M}$ corresponding to $x$ and $B \cong A[u] / p(u)$ where $p(u)=$ $u^{3}+\alpha u^{2}+\beta u+\gamma, \alpha, \beta, \gamma \in A$. We can also assume that $y$ corresponds to the ideal $(u)+\mathfrak{M}$ of $B$. In this setting $R_{\sigma}$ has equations $3 u^{2}+2 \alpha u+\beta=p(u)=0$. It follows that $y \in R_{\sigma}$ if and only if $\beta, \gamma \in \mathfrak{M}$. Moreover $\alpha \notin \mathfrak{M}$ since $x$ is not a point of total ramification of $\sigma$. An easy local computation shows that $\gamma \notin \mathfrak{M}^{2}$, otherwise $S$ would be singular at $y$. Eliminating the variable $u$ we finally obtain an equation of $B_{\sigma}$ of the form $b:=4 \alpha^{3} \gamma+b_{2}$ where $b_{2} \in \mathfrak{M}^{2}$.

Since $\operatorname{spec}(B) \cong \mathbb{A}_{A}^{\prime} \subseteq \mathbb{P}_{A}^{1}$ and $\mathcal{O}_{\mathrm{P}_{A}^{\prime}}(1)_{\text {|spec }(B)} \cong \omega_{\text {spec }(B) \mid \operatorname{spec}(A)}$ (see the introduction) and $\left|\omega_{s \mid \mathbb{P}_{\mathbf{C}}^{2}}\right|=\left|C_{0}+e^{*}(P+3 Q)\right|$, a proper choice of $u$ allows us to assume that $u=p(u)=0$ are equations of $C_{0}$, thus $B_{\tau} \in\left|4 C_{0}-2 e^{*} Q\right|$ is given by $p(u)=q(u)=0$, where $q(u)$ is a polynomial of degree 4 . It follows that we can choose equations $p(u)=\delta u^{2}+\varepsilon u+\eta=$ $0, \delta, \varepsilon, \eta \in A$, for $B_{\tau}$. The condition $y \in B_{\tau}$ yields $\eta \in \mathfrak{M}$. Again eliminating the variable $u$ we obtain the equation of $C_{\ell}$ of the form $c=\left(\alpha \delta \varepsilon-\varepsilon^{3}\right) \gamma+c_{2}$ where $c_{2} \in \mathfrak{M}^{2}$.

Theorem 2.6. $\quad C_{e}$ is an irreducible sextic birationally isomorphic to E. Its singularities are either nine points of type $A_{1}$, possibly three by three infinitely near (i.e. three points of type $D_{4}$ ), or nine cusps of type $A_{2}$.

Each cuspidal tangent lines at $B_{\sigma}$ contains exactly one singular point of $C_{e} . C_{e}$ has points of type $D_{4}$ if and only if $\operatorname{Sing}\left(C_{Q}\right)=O_{i}$ for some $i=0,1,2,3 . C_{Q}$ has points of type $A_{2}$ if and only if $C_{Q}=B_{\sigma}$ and, in this case, $\varrho$ is the cover described in Example 1.7.

Finally $C_{Q}$ and $B_{\sigma}$ are tangent at each point of intersection.

Proof. If $C_{\boldsymbol{e}} \cap \operatorname{Sing}\left(B_{\sigma}\right) \neq \emptyset$ then $B_{\tau}$ contains at least one point of total ramification of $\sigma$, whence $R_{0} \cap B_{\tau} \neq \emptyset$. Since $R_{0} \cdot B_{\tau}=0$ we get that $R_{0}=B_{\tau}$, hence $C_{Q}=B_{\sigma}$ and $\varrho$ is the cover described in Example 1.7.

For this reason, from now on, we will always assume that $B_{\tau} \neq R_{0}$, i.e. $C_{Q} \cap \operatorname{Sing}\left(B_{\sigma}\right)=\emptyset$. Notice that it follows from Remark 2.5 that $C_{Q}$ and $B_{\sigma}$ are tangent at each point of intersection.

Since $B_{\tau}$ is irreducible and $B_{\tau} \cdot\left(C_{0}+e^{*} Q\right)=6$ then $C_{\ell}$ is an irreducible sextic curve. If $C_{Q}$ was not reduced then its reduced structure $\left(C_{Q}\right)_{\text {red }}$ would be either a conic or a cubic, thus $B_{\tau} \subseteq \sigma^{-1}\left(\left(C_{Q}\right)_{r e d}\right) \in\left|n\left(C_{0}+e^{*} Q\right)\right|$, where $n=2,3$, which is absurd since $B_{\tau} \in\left|4 C_{0}-2 e^{*} P\right|$.

It follows that $\sigma_{\mid B_{\tau}}: B_{\tau} \rightarrow C_{Q}$ is a resolution of singularities of $C_{\ell}$, which is then birationally isomorphic to $E$. Therefore the formula of Clebsch becomes

$$
\sum_{x \in C_{e}} \frac{m_{x}\left(m_{x}-1\right)}{2}=9
$$

where $m_{x}$ is the multiplicity of $x$. We also obtain that if the tangent lines at $x \in \operatorname{Sing}\left(C_{\ell}\right)$ are not all distinct, then $x \in B_{\sigma}$. Since $C_{\ell}$ is $K(D)$-invariant, we get that $\operatorname{Sing}\left(C_{Q}\right)$ is union of $K(D)$-orbits.

Assume that $\operatorname{Sing}\left(C_{\ell}\right)$ contains either a point of type $A_{k}$, with $k \geq 3$, or a nonordinary point of multiplicity at least three. Such kind of points contribute at least two 
in the sum in formula 2.6.1. Then there is $i=0,1,2,3$ such that $\emptyset \neq \operatorname{Sing}\left(C_{\ell}\right) \cap O_{i} \subseteq$ $B_{\sigma} \cap O_{i}=\emptyset$, a contradiction.

If $\operatorname{Sing}\left(C_{\rho}\right)$ contains an ordinary multiple point $x$ of multiplicity at least three, 2.6.1 implies that $x$ must be of type $D_{4}$ and $x \in O_{i}$, thus $\operatorname{Sing}\left(C_{\ell}\right)=O_{i}$ for some $i=0,1,2,3$. Conversely if $a=0$ in equation 2.2 then $O_{0} \subseteq \operatorname{Sing}\left(C_{e}\right)$. If also $c=0$ then equality holds and each point is of type $D_{4}$. If $c \neq 0$ then the points of $O_{0}$ are of type $A_{1}$. Then by 2.6.1 we necessarily have $\operatorname{Sing}\left(C_{e}\right)=\cup O_{i}$. As proved above the points in $O_{i}$ must be ordinary, hence again by 2.6 .1 they are all of type $A_{1}$ and $\operatorname{Sing}\left(C_{Q}\right)=O_{0} \cup O_{i} \cup O_{j}$ $(i, j=1,2,3, i \neq j)$.

Now let the singularities of $C_{Q}$ be nine points of type $A_{2}$. Then the equation of $C_{Q}$ is $f_{\mu}$ (see formula 2.3), hence $\operatorname{Sing}\left(C_{\varrho}\right)=O\left([\mu, 1,1]\right.$ ). Moreover $C_{\varrho}$ is birationally isomorphic to $E$. Thus we can suppose that either $\lambda^{3}=\mu^{3}$ or $\mu=(\lambda+2) /(\lambda-1)$ by remark 2.4. Moreover $\operatorname{Sing}\left(C_{\ell}\right) \subseteq B_{\sigma}$, since $\varrho$ is locally étale outside $B_{\sigma}$.

If $\lambda=\mu=0$ then $C_{Q}=B_{\sigma}$. Assume that $\lambda \mu \neq 0$ and $\lambda^{3}=\mu^{3}$. Since $C_{Q} \cdot B_{\sigma}=36$, Remark 2.5 implies that the pencil $\Phi$ of sextic curves generated by $C_{e}$ and $B_{\sigma}$ has at most 18 base points. On the other hand $\Phi$ contains a reducible curve $\bar{C}$ of equation $x_{0} x_{1} x_{2}\left(x_{0}^{3}+x_{1}^{3}+x_{2}^{3}-3 m x_{0} x_{1} x_{2}\right)=0$. It is not difficult to check that $\bar{C} \cap B_{\sigma}$ contains at least 27 points, which are base points of $\Phi$.

Assume finally that $\mu=(\lambda+2) /(\lambda-1)$. By direct substitution one checks that the condition $\operatorname{Sing}\left(C_{Q}\right) \subseteq B_{\sigma}$ is equivalent to $\operatorname{Sing}\left(B_{\sigma}\right) \subseteq C_{\ell}$. Thus in both these cases we obtain a contradiction.

The point $\bar{x}:=\left[\bar{x}_{0}, \bar{x}_{1}, \bar{x}_{2}\right] \in \mathbb{P}_{\mathrm{C}}^{2}$ is singular on the curve $C$ of equation 2.2 , if and only if $[a, b, c, d] \in \mathbb{P}_{\mathbf{C}}^{3}$ is a solution of the homogeneous system

$$
\left\{\begin{array}{l}
6 \bar{x}_{0}^{5} a+3 \bar{x}_{0}^{2}\left(\bar{x}_{1}^{3}+\bar{x}_{2}^{3}\right) b+\bar{x}_{1} \bar{x}_{2}\left(4 \bar{x}_{0}^{3}+\bar{x}_{1}^{3}+\bar{x}_{2}^{3}\right) c+2 \bar{x}_{0} \bar{x}_{1}^{2} \bar{x}_{2}^{2} d=0 \\
6 \bar{x}_{1}^{5} a+3 \bar{x}_{1}^{2}\left(\bar{x}_{0}^{3}+\bar{x}_{2}^{3}\right) b+\bar{x}_{0} \bar{x}_{2}\left(\bar{x}_{0}^{3}+4 \bar{x}_{1}^{3}+\bar{x}_{2}^{3}\right) c+2 \bar{x}_{0}^{2} \bar{x}_{1} \bar{x}_{2}^{2} d=0 \\
6 \bar{x}_{2}^{5} a+3 \bar{x}_{2}^{2}\left(\bar{x}_{0}^{3}+\bar{x}_{1}^{3}\right) b+\bar{x}_{0} \bar{x}_{1}\left(\bar{x}_{0}^{3}+\bar{x}_{1}^{3}+4 \bar{x}_{2}^{3}\right) c+2 \bar{x}_{0}^{2} \bar{x}_{1}^{2} \bar{x}_{2} d=0 .
\end{array}\right.
$$

Let us denote by $M$ the matrix of the system 2.6.2.

Obviously the system 2.6 .2 has always $\infty^{\prime}$ solutions, corresponding to the unique curve of equation $\left(x_{0}^{3}+x_{1}^{3}+x_{2}^{3}-3 m x_{0} x_{1} x_{2}\right)^{2}=0$ passing through $\bar{x}$.

Generically $\operatorname{rk}(M)=3$. In order to have also solutions representing irreducible curves, we need $\operatorname{rk}(M) \leq 2$. Some easy computations show that the ideal $I$ of $3 \times 3$-minors of $M$ is generated by the three polynomials

$$
\bar{x}_{0}^{2} \bar{x}_{1}^{2} \bar{x}_{2}^{2} q(\bar{x}), \quad \bar{x}_{0} \bar{x}_{1} \bar{x}_{2}\left(\bar{x}_{0}^{3}+\bar{x}_{1}^{3}+\bar{x}_{2}^{3}\right) q(\bar{x}), \quad\left(\bar{x}_{0}^{3}+\bar{x}_{1}^{3}+\bar{x}_{2}^{3}\right)^{2} q(\bar{x}),
$$

where $q(\bar{x}):=\left(\bar{x}_{0}^{3}-\bar{x}_{1}^{3}\right)\left(\bar{x}_{0}^{3}-\bar{x}_{2}^{3}\right)\left(\bar{x}_{1}^{3}-\bar{x}_{2}^{3}\right)$. It follows that $I \subseteq(q)$.

On the other hand if $\bar{x} \in \mathbb{P}_{C}^{2}$ is singular on $C$ but $q(\bar{x}) \neq 0$, then $\bar{x}_{0} \bar{x}_{1} \bar{x}_{2}=0$. If $\bar{x}_{0}=0$, we get $\bar{x}_{1}^{3}+\bar{x}_{2}^{3}=0$. Assuming $\bar{x}_{1}=1$ and $\bar{x}_{2}=-1$, then 2.6 .2 becomes $2 a-b=0$. If $a=0$ then $C$ is reducible. Assume $a=1$, then 2.2 becomes

$$
\left(x_{0}^{3}+x_{1}^{3}+x_{2}^{3}\right)^{2}+c x_{0} x_{1} x_{2}\left(x_{0}^{3}+x_{1}^{3}+x_{2}^{3}\right)+d x_{0}^{2} x_{1}^{2} x_{2}^{2}=0
$$

which is reducible too. 
We have proved that $\bar{x} \in \operatorname{Sing}\left(C_{\ell}\right)$ if and only if $q(\bar{x})=0$. Notice that $q(x)=0$ is the equation of the union of the cuspidal tangent lines at $B_{\sigma}$ and it is easy to check that each cuspidal tangent line contains a singular point of $C_{e}$.

\section{The sheaves $\mathcal{E}$ and $\mathcal{F}$}

In this section we prove Theorem 0.4. Let $(A,|D|)$ be a $(1,3)$-polarized abelian surface satisfying $(\mathcal{O})$. One has for $n \geq 1$

$$
h^{i}\left(A, \mathcal{O}_{A}(n D)\right)= \begin{cases}3 n^{2} & \text { if } i=0, \\ 0 & \text { if } i=1,2 .\end{cases}
$$

Since $K_{A} \sim 0$ then, by adjuction, $p_{a}(D)=4$. If $C \in|D|$ is smooth then the map $\varrho_{1 C}: C \rightarrow \mathbb{P}_{C}^{1}$ is branched at 18 points hence $\varrho$ is branched along a curve of degree 18 . Finally

$$
\omega_{A \mid \mathbb{P}_{\mathrm{C}}^{2}} \cong \omega_{A \mid \mathrm{C}} \otimes \varrho^{*} \omega_{\mathbb{C}_{C}^{2} \mid \mathrm{C}}^{-1} \cong \varrho^{*} \mathcal{O}_{\mathbb{P}_{\mathrm{C}}^{2}}(3) \cong \mathcal{O}_{A}(3 D) .
$$

As usual one has the isomorphisms 0.2. Since

$$
h^{i}\left(A, \mathcal{O}_{A}(n D)\right)=h^{i}\left(A, \varrho^{*} \mathcal{O}_{\mathbb{P}_{\mathbf{C}}^{2}}(n)\right)=h^{i}\left(\mathbb{P}_{\mathbf{C}}^{2}, \mathcal{O}_{\mathbb{P}_{\mathbf{C}}^{2}}(n)\right)+h^{i}\left(\mathbb{P}_{\mathbf{C}}^{2}, \check{\mathcal{E}}(n)\right),
$$

using 3.1, Bott's formulas and Serre duality one easily checks that

$$
h^{i}\left(\mathbb{P}_{\mathrm{C}}^{2}, \check{\mathcal{E}}(n)\right)=h^{i}\left(\mathbb{P}_{\mathrm{C}}^{2}, \mathcal{O}_{\mathbb{P}_{\mathrm{C}}^{2}}(n-3)\right)+2 h^{i}\left(\mathbb{P}_{\mathrm{C}}^{2}, \Omega_{\mathrm{P}_{\mathrm{C}}^{2} \mathrm{C}}^{1}(n)\right),
$$

for every $n \in \mathbb{Z}$ and $i=0,1,2$.

Lemma 3.3. Let $\mathcal{H}$ be a locally free $\mathcal{O}_{\mathbb{P}^{2}}$-sheaf of rank 4 such that

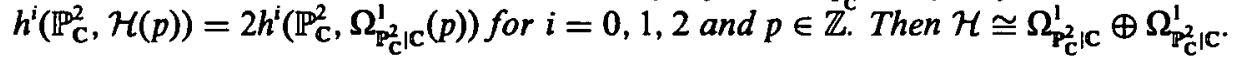

Proof. The only non-zero terms in the Beilinson's spectral sequence (see [16]) are $E_{1}^{-2,2} \cong \mathcal{O}_{\mathbb{P}_{\mathrm{c}}^{2}}^{\oplus 2}$ and $E_{1}^{0,1} \cong \mathcal{O}_{\mathrm{P}_{\mathrm{c}}^{2}}(-1)^{\oplus 6}$. It follows $E_{1}^{0,1} \cong E_{2}^{0,1}$ and $E_{1}^{-2,2} \cong E_{2}^{-2,2}$, hence a complex

$$
0 \rightarrow E_{2}^{-2,2} \stackrel{d_{2}^{-2,2}}{\rightarrow} E_{2}^{0,1} \rightarrow 0
$$

is defined. Moreover $E_{r}^{0,1} \cong E_{3}^{0,1}$ and $E_{r}^{-2,2} \cong E_{3}^{-2,2}$ for any $r \geq 3$. Since $E_{\infty}^{0,1}=0$ then $d_{2}^{-2,2}$ is surjective. On the other hand $E_{\infty}^{-2,2} \cong \mathcal{H}$ hence the complex 3.3.1 yields the following exact sequence

$$
0 \rightarrow \mathcal{H} \rightarrow \mathcal{O}_{\mathrm{P}_{\mathrm{C}}^{2}}(-1)^{\oplus 6} \stackrel{s}{\rightarrow} \mathcal{O}_{\mathrm{P}_{\mathrm{C}}^{2}}^{\oplus 2} \rightarrow 0 .
$$

The matrix $S$ of $s$ is of the form 


$$
S=\left(\begin{array}{llllll}
a_{0} & a_{1} & a_{2} & a_{3} & a_{4} & a_{5} \\
b_{0} & b_{1} & b_{2} & b_{3} & b_{4} & b_{5}
\end{array}\right)
$$

where $a_{i}, b_{i} \in H^{0}\left(\mathbb{P}_{C}^{2}, \mathcal{O}_{\mathbb{P}_{C}^{2}}(1)\right)$. Since $\operatorname{rk}(S)=2$ then $H^{0}\left(\mathbb{P}_{C}^{2}, \mathcal{O}_{\mathbb{P}_{C}^{2}}(1)\right)$ is generated by the $a_{i}$ 's, otherwise there exists a point $x \in \mathbb{P}_{\mathrm{C}}^{2}$ such that $\operatorname{rk}\left(s_{x}\right) \leq 1$. In particular, up to a proper choice of a basis of $\mathcal{O}_{\mathrm{P}_{\mathrm{c}}^{2}}(-1)^{\oplus 6}$ which corresponds to a proper sequence of elementary operations on the columns of $S$, one can assume that

$$
S=\left(\begin{array}{llllll}
x_{0} & x_{1} & x_{2} & 0 & 0 & 0 \\
b_{0} & b_{1} & b_{2} & b_{3} & b_{4} & b_{5}
\end{array}\right) \text {. }
$$

If $b_{3}, b_{4}, b_{5}$ were linearly dependent then $\mathcal{H}$ would contain $\mathcal{O}_{\mathbb{P}_{c}^{2}}(-1)$ as direct summand. Hence

$$
0 \neq h^{0}\left(\mathbb{P}_{\mathrm{C}}^{2}, \mathcal{H}(1)\right)=2 h^{0}\left(\mathbb{P}_{\mathbf{C}}^{2}, \Omega_{\mathbb{C}_{\mathbf{C}}^{2} \mathbf{C}}^{1}(1)\right)=0 .
$$

We conclude that, up to a proper choice of a basis of $\mathcal{O}_{p_{c}^{2}}(-1)^{\oplus 6}$, one gets

$$
S=\left(\begin{array}{cccccc}
x_{0} & x_{1} & x_{2} & 0 & 0 & 0 \\
0 & 0 & 0 & x_{0} & x_{1} & x_{2}
\end{array}\right)
$$

hence $\mathcal{H} \cong \Omega_{\mathrm{P}_{\mathbf{C}}^{2} \mid \mathrm{C}}^{1} \oplus \Omega_{\mathrm{P}_{\mathrm{C}}^{2} \mid \mathrm{C}}^{1}$.

Proof of isomorphism 0.4.1. The isomorphism

$$
\mathcal{O}_{\mathrm{P}_{\mathrm{C}}^{2}} \oplus \mathcal{E} \cong \varrho_{*} \omega_{A \mid \mathrm{P}_{\mathrm{C}}^{2}} \cong \varrho_{*} \varrho^{*} \mathcal{O}_{\mathrm{P}_{\mathrm{C}}^{2}}(3) \cong \mathcal{O}_{\mathrm{P}_{\mathrm{C}}^{2}}(3) \oplus \check{\mathcal{E}}(3) \text {, }
$$

gives rise to a factorization of the identity on $\mathcal{O}_{\mathrm{P}_{\mathrm{C}}^{2}}$ as

$$
\mathcal{O}_{\mathbf{P}_{\mathbf{C}}^{2}} \stackrel{i}{\rightarrow} \mathcal{O}_{\mathbb{P}_{\mathbf{C}}^{2}}(-3) \oplus \mathcal{E}(-3) \stackrel{p}{\rightarrow} \mathcal{O}_{\mathbb{P}_{\mathbf{C}}^{2}}
$$

Since $h^{0}\left(\mathbb{P}_{\mathrm{C}}^{2}, \mathcal{O}_{\mathbb{P}_{\mathrm{C}}^{2}}(-3)\right)=0$ one can split both $i$ and $p$ through $\mathcal{E}(-3)$ hence $\mathcal{E} \cong \mathcal{O}_{\mathbf{P}_{\mathrm{C}}^{2}}(3) \oplus \mathcal{E}_{0}$. Thus identities 3.2 imply that

$$
h^{i}\left(\mathbb{P}_{\mathbf{C}}^{2}, \check{\mathcal{E}}_{0}(n)\right)=2 h^{i}\left(\mathbb{P}_{\mathbf{C}}^{2}, \Omega_{\mathbf{P}_{\mathbf{C}}^{2} \mid \mathbf{C}}^{1}(n)\right), \quad i=0,1,2, n \in \mathbb{Z} .
$$

It follows from Lemma 3.3 that $\check{\mathcal{E}}_{0} \cong \Omega_{\mathrm{P}_{\mathrm{C}}^{2} \mathrm{C}}^{1} \oplus \Omega_{\mathrm{P}_{\mathrm{C}}^{2} \mid \mathrm{C}}^{1}$.

Proof of isomorphism 0.4.2. Consider the exact sequence 0.3. Since $\omega_{A \mid C} \cong \mathcal{O}_{A}$ then $\omega_{A \mid \mathbb{R}_{C}^{2}} \cong \varrho^{*} \mathcal{O}_{p_{C}^{2}}(3)$. Thus, taking into account the decomposition of $\mathcal{E}$ and the

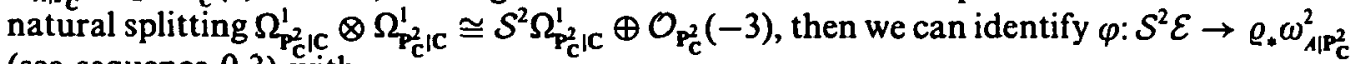
(see sequence 0.3 ) with

$$
\varphi: \mathcal{O}_{\mathrm{P}_{\mathrm{C}}^{2}}(3) \oplus \Omega_{\mathrm{P}_{\mathrm{C}}^{2} \mid \mathrm{C}}^{1}(6)^{\oplus 2} \oplus \mathcal{O}_{\mathrm{P}_{\mathrm{C}}^{2}}(6) \oplus \mathcal{S}^{2} \Omega_{\mathrm{P}_{\mathrm{C}}^{2} \mathrm{C}}^{1}(6)^{\oplus 3} \rightarrow \mathcal{O}_{\mathrm{P}_{\mathrm{C}}^{2}}(3) \oplus \Omega_{\mathrm{P}_{\mathrm{C}} \mid \mathrm{C}}^{1}(6)^{\oplus 2} \oplus \mathcal{O}_{\mathrm{P}_{\mathrm{C}}^{2}}(6) .
$$

We want to prove that $\varphi$ has a section. To this purpose note that $\varphi$ induces two 
morphisms

$$
\begin{aligned}
& \varphi_{1}: \mathcal{O}_{\mathbb{P}_{\mathrm{C}}^{2}}(3) \oplus \Omega_{\mathrm{P}_{\mathrm{C}}^{2} \mid \mathrm{C}}^{1}(6)^{\oplus 2} \oplus \mathcal{O}_{\mathrm{P}_{\mathrm{C}}^{2}}(6) \rightarrow \mathcal{O}_{\mathrm{P}_{\mathrm{C}}^{2}}(3) \oplus \Omega_{\mathrm{P}_{\mathrm{C}}^{2} \mid \mathrm{C}^{1}}^{1}(6)^{\oplus 2} \oplus \mathcal{O}_{\mathrm{P}_{\mathrm{C}}^{2}}(6), \\
& \varphi_{2}: \mathcal{S}^{2} \Omega_{\mathrm{P}_{\mathrm{C}}^{2} \mid \mathrm{C}}^{1}(6)^{\oplus 3} \rightarrow \mathcal{O}_{\mathrm{P}_{\mathrm{C}}^{2}}(3) \oplus \Omega_{\mathrm{P}_{\mathrm{C}}^{2} \mid \mathrm{C}}^{1}(6)^{\oplus 2} \oplus \mathcal{O}_{\mathrm{P}_{\mathrm{C}}^{2}}(6) .
\end{aligned}
$$

There exists a natural monomorphism $i: \varrho_{*} \omega_{A \mathbb{R}_{C}^{2}}^{2} \rightarrow \mathcal{S}^{2} \mathcal{E}$ such that $\varphi \circ i=\varphi_{1}$. We now prove that $\varphi_{1}$ is an isomorphism. Let $r \subseteq \mathbb{P}_{\mathbf{C}}^{2}$ be a line. We claim that $\varphi_{1 \mid r}$ is an isomorphism. If this is the case then

$$
\operatorname{det}\left(\varphi_{1}\right) \in H^{0}\left(\mathbb{P}_{\mathbf{C}}^{2}, \operatorname{det}\left(\varrho_{*} \omega_{A \mid \mathbb{P}_{\mathbf{C}}^{2}}^{2}\right) \otimes \operatorname{det}\left(\varrho_{*} \omega_{A \mid \mathbb{P}_{C}^{2}}^{2}\right)^{-1}\right) \cong \mathbb{C} .
$$

Since, by the claim, $\operatorname{det}\left(\varphi_{1 \mid r}\right) \neq 0$ then $\operatorname{det}\left(\varphi_{1}\right) \neq 0$ too. Let $\psi:=i \circ \varphi_{1}^{-1} \cdot \psi$ is a section of $\varphi$ hence

$$
\mathcal{F} \cong \mathcal{S}^{2} \mathcal{E} / \mathrm{im}(\psi) \cong\left(\mathcal{S}^{2} \Omega_{\mathrm{P}_{\mathrm{C}}^{2} \mathbf{C}}^{1}(6)\right)^{\oplus 3}
$$

Now we prove the claim. Assume that $C_{r}:=\varrho^{*} r$ is smooth: set $\varrho_{r}:=\varrho_{\mid r}, \mathbb{P}_{r}:=\pi^{-1}(r)$, $\mathcal{E}_{r}:=\mathcal{E}_{\mid r}$, fix an identification $\Omega_{\mathbb{P}_{\mathrm{C}}^{2} \mid \mathrm{C}}^{1}(6)_{i r} \cong \mathcal{O}_{r}(4) \oplus \mathcal{O}_{r}(5)$ and take non-zero sections

$$
\begin{aligned}
s, t & \in H^{0}\left(\mathbb{P}_{r}, \mathcal{O}_{\mathbf{P}_{r}} \otimes \varrho_{r}^{*} \mathcal{O}_{r}(-1)\right) \cong H^{0}\left(r, \mathcal{E}_{r}(-1)\right), \\
v, w & \in H^{0}\left(\mathbb{P}_{r}, \mathcal{O}_{\mathbb{P}_{r}} \otimes \varrho_{r}^{*} \mathcal{O}_{r}(-2)\right) \cong H^{0}\left(r, \mathcal{E}_{r}(-2)\right), \\
u & \in H^{0}\left(\mathbb{P}_{r}, \mathcal{O}_{\mathbb{P}_{r}} \otimes \varrho_{r}^{*} \mathcal{O}_{r}(-3)\right) \cong H^{0}\left(r, \mathcal{E}_{r}(-3)\right) .
\end{aligned}
$$

The matrices $M$ of $\varphi_{i r}$ and $M_{i}$ of $\varphi_{i \mid r}$ satisfy $M=\left(M_{1} \mid M_{2}\right)$. Moreover

$$
M_{1}=\left(\begin{array}{cccccc}
a & 0 & 0 & 0 & 0 & 0 \\
b_{1} & c_{1,1} & c_{1,2} & 0 & 0 & 0 \\
b_{2} & c_{2,1} & c_{2,2} & 0 & 0 & 0 \\
d_{1} & e_{1,1} & e_{1,2} & f_{1,1} & f_{1,2} & 0 \\
d_{2} & e_{2,1} & e_{2,2} & f_{2,1} & f_{2,2} & 0 \\
g & h_{1} & h_{2} & m_{1} & m_{2} & n
\end{array}\right)
$$

whose elements have degrees

$$
\left(\begin{array}{cccccc}
0 & -1 & -1 & -2 & -2 & -3 \\
1 & 0 & 0 & -1 & -1 & -2 \\
1 & 0 & 0 & -1 & -1 & -2 \\
2 & 1 & 1 & 0 & 0 & -1 \\
2 & 1 & 1 & 0 & 0 & -1 \\
3 & 2 & 2 & 1 & 1 & 0
\end{array}\right) .
$$


The first row of $M$ is the matrix associated to $\vartheta_{r}: \mathcal{S}^{2} \mathcal{E}_{r} \rightarrow \mathcal{O}_{r}(3)$.

It follows that if $a=0$, then the first row of $M$ would be 0 which is absurd since $\varphi$ is surjective.

If $a \neq 0$ and $\operatorname{rk}\left(M_{1}\right) \leq 5$, then the system $M x=0$ has a solution $\bar{x}:=$ $\left(0, \alpha_{2}, \ldots, \alpha_{6}, 0, \ldots, 0\right) \neq 0$ hence

$$
C_{r} \subseteq Q=V_{+}\left(u\left(\alpha_{2} s+\alpha_{3} t+\alpha_{4} v+\alpha_{5} w+\alpha_{6} u\right)\right) \subseteq \mathbb{P}_{r}
$$

Since $C_{r}$ is irreducible this is absurd.

Hence the claim is proved.

Remark 3.4. It is not difficult to check that if a section $\eta \in H^{0}\left(\mathbb{P}_{\mathbf{C}}^{2}, \check{\mathcal{F}} \otimes \mathcal{S}^{2} \mathcal{E}\right)$ defines the smooth surface $A:=D_{0}\left(\Phi_{6}(\eta)\right) \subseteq \mathbb{P}$ then $A$ is an abelian surface and $\varrho:=\pi_{1 A}$ is a cover of degree 6.

Unfortunately, by dimensional reasons, the generic section $\eta$ does not define a surface.

\section{Bielliptic abelian surfaces in $\mathbb{P}(\mathcal{E})$}

In this last section we characterize $(1,3)$-polarized bielliptic abelian surfaces with respect to the behaviour of the embedding $i: A \hookrightarrow \mathbb{P}(\mathcal{E})$.

Let $(A,|D|)$ be a $(1,3)$-polarized abelian surface satisfying condition $(\mathcal{O})$. Let $\varrho: A \rightarrow \mathbb{P}_{\mathrm{C}}^{2}$ be the corresponding cover of degree 6 . It follows from the previous section and Theorem 2.1 of [9] applied to $\varrho$ the existence of a unique embedding $i: A \hookrightarrow \mathbb{P}$ such that $\varrho=\pi \circ i$ and the scheme-theoretic fibre $A_{y}:=\varrho^{-1}(y) \subseteq \mathbb{P}_{k(y)}^{4} \cong \mathbb{P}_{y}:=\pi^{-1}(y)$ is an arithmetically Gorenstein subscheme.

Moreover such embedding is induced by the composition of $\varrho^{*} \mathcal{E} \hookrightarrow \varrho^{*}\left(\mathcal{O}_{\mathbf{P}_{\mathbf{C}}^{2}} \oplus \mathcal{E}\right) \stackrel{\sim}{\longrightarrow}$ $\varrho^{*} \varrho_{*} \omega_{A \mathbb{P}_{C}^{2}}$, see 0.2 , followed by $\varrho^{*} \varrho_{*} \omega_{A \| \mathbb{P}_{C}^{2}} \rightarrow \omega_{A \mathbb{P}_{C}^{2}}$.

We fix a decomposition $\mathcal{E} \cong \mathcal{O}_{\mathrm{P}_{\mathrm{C}}^{2}}(3) \oplus \Omega_{\mathrm{P}_{\mathrm{C}} \mid \mathrm{C}}^{1}(3) \oplus \Omega_{\mathrm{P}_{\mathrm{C}} \mid \mathrm{C}}^{1}(3)$. The two projections (on the sum of the first two summands and on the third one), allow us to define two subbundles $\mathbb{P}\left(\Omega_{\mathrm{p}_{\mathrm{C}}^{2} \mid \mathrm{C}}^{1}(3)\right) \cong U \subseteq \mathbb{P}$ and $\mathbb{P}\left(\mathcal{O}_{\mathrm{P}_{\mathrm{C}}^{2}}(3) \oplus \Omega_{\mathrm{P}_{\mathrm{C}}^{2} \mathrm{C}}^{1}(3)\right) \cong V \subseteq \mathbb{P}$. Let $\bar{\pi}: \mathbb{P} \rightarrow U$ be the projection from $V$.

Let $S$ be the closure of $\bar{\pi}(A)$. There exists a dominant rational map $\tau: A \rightarrow S$ and we define $\sigma:=\pi_{\mid S}$, so that $\varrho=\sigma \circ \tau$. Since $S \subseteq U$ is a divisor then it is locally Gorenstein, hence $\sigma$ is a Gorenstein cover.

Since $\operatorname{deg}(\varrho)=6$ then $\operatorname{deg}(\tau)=1,2,3,6$, and if $\operatorname{deg}(\tau)=6$ then $\operatorname{deg}(\sigma)=1$ and the map $\mathcal{O}_{\mathrm{P}_{\mathrm{C}}^{2}} \rightarrow \sigma_{*} \mathcal{O}_{s}$ is an isomorphism, thus the same is true for $\sigma$. If $x \in S$ is a general point then $\varrho^{-1}(\sigma(x))=\tau^{-1}(x) \subseteq\left\langle x, V \cap \pi^{-1}(x)\right\rangle \cong \mathbb{P}_{k(x)}^{3} \subseteq \pi^{-1}(x)$, which is absurd since $\varrho^{-1}(\sigma(x)) \subseteq \pi^{-1}(x)$ is arithmetically Gorenstein (see [9, Theorem 2.1] and [19, Lemma 4.2]).

Proposition 4.1. Let $S$ be smooth and assume that $\tau$ is a morphism. Then $A$ is bielliptic and the maps $\sigma$ and $\tau$ coincide with the ones defined in Propositions 1.1 and 1.5 respectively. 
Proof. Since $\operatorname{deg}(\varrho)=6$ then $\operatorname{deg}(\tau)=1,2,3,6$ and the case $\operatorname{deg}(\tau)=6$ is impossible as shown above. Moreover the smoothness of $S$ yields that $\tau$ is actually a cover.

If $\operatorname{deg}(\tau)=1$ the map $\mathcal{O}_{S} \rightarrow \tau_{*} \mathcal{O}_{A}$ is an isomorphism, thus the same is true for $\tau$. The surjective map $\mathcal{O}_{\mathrm{P}_{\mathrm{C}}^{2}}(1)^{\oplus 3} \rightarrow \Omega_{\mathrm{P}_{\mathrm{C}}^{2} \mathrm{C}}^{1}(3)$, yields

$$
A \subseteq \mathbb{P}\left(\Omega_{\mathbf{P}_{\mathbf{C}}^{2} \mid}^{1}(3)\right) \subseteq \mathbb{P}\left(\mathcal{O}_{\mathbf{P}_{\mathbf{C}}^{2}}(1)^{\oplus 3}\right) \cong X:=\mathbb{P}_{\mathbf{C}}^{2} \times \mathbb{P}_{\mathbf{C}}^{2}
$$

Let $p_{i}: X \rightarrow \mathbb{P}_{\mathrm{C}}^{2}$ be the projection onto the $i$-th factor and as usual set $\mathcal{O}_{X}(a, b):=p_{1}^{*} \mathcal{O}_{\mathbb{P}_{\mathrm{C}}^{2}}(a) \otimes p_{2}^{*} \mathcal{O}_{\mathbb{P}_{\mathrm{C}}^{2}}(b)$. If $h_{1}$ and $h_{2}$ are the classes of $\mathcal{O}_{X}(1,0)$ and $\mathcal{O}_{X}(0,1)$ respectively in the Chow ring $A(X)$, then there are $\alpha, \beta, \gamma \in \mathbb{Z}$ such that the class of $A$ is $\alpha h_{1}^{2}+\beta h_{2}^{2}+\gamma h_{1} \cdot h_{2}$. It is proved in Section 2 of [12], that $\alpha=6, \beta=0$, hence $\gamma$ is a solution of $\gamma^{2}-9 \gamma-18=0$ which has not integral solutions.

Thus $\operatorname{deg}(\tau)=2,3$. Assume that $\operatorname{deg}(\tau)=3$ and let $\mathcal{O}_{P_{c}^{2}}(n)$ and $\mathcal{F}$ be the Tschirnhausen modules of $\sigma$ and $\tau$ respectively. Since, in this case, $B_{Q}=3 B_{\sigma}+\sigma_{*} B_{\tau}$ then $\operatorname{deg}\left(B_{\sigma}\right) \leq 6$, hence $n=-1,-2,-3$. From the isomorphisms

$$
\mathcal{O}_{P_{\mathrm{C}}^{2}} \oplus \check{\mathcal{E}} \cong \varrho_{*} \mathcal{O}_{A} \cong \sigma_{*} \tau_{*} \mathcal{O}_{A} \cong \mathcal{O}_{\mathbf{P}_{\mathrm{C}}^{2}} \oplus \mathcal{O}_{\mathbf{P}_{\mathrm{C}}^{2}}(n) \oplus \sigma_{*} \mathcal{F}
$$

and formula 0.4 .1 , we obtain a factorization of the identity

$$
\mathcal{O}_{\mathbf{P}_{\mathrm{C}}^{2}}(n) \nrightarrow \mathcal{O}_{\mathbb{P}_{\mathrm{C}}^{2}} \oplus \mathcal{O}_{\mathbf{P}_{\mathrm{C}}^{2}}(-3) \oplus \Omega_{\mathbf{P}_{\mathrm{C}}^{2} \mid \mathrm{C}}^{1} \oplus \Omega_{\mathbf{P}_{\mathrm{C}}^{2} \mid \mathrm{C}}^{1} \rightarrow \mathcal{O}_{\mathbf{P}_{\mathrm{C}}^{2}}(n)
$$

On the other hand $h^{0}\left(\mathbb{P}_{\mathbf{C}}^{2}, \Omega_{\mathbb{P}_{C}^{2} \mid \mathrm{C}}^{1}(1)\right)=0$, thus only the case $n=-3$ is possible. In this case $S$ is a $K 3$ surface hence $\tau$ is étale. Therefore $0=\chi\left(\mathcal{O}_{A}\right)=3 \chi\left(\mathcal{O}_{s}\right)=6$ (see [15] or [17]).

Assume now that $\operatorname{deg}(\tau)=2$. Fix a line $\ell \in \mathbb{P}_{C}^{2}$ such that both $E:=\sigma^{-1}(\ell)$ and $C:=\varrho^{-1}(\ell)$ are smooth. Let $p$ be the geometric genus of $E$ and define $t:=\tau_{\mid C}, s:=\Sigma_{\mid E}$, $r:=\varrho_{\mid c}$. The branch loci of $r, s, t$ satisfy $B_{r}=2 B_{s}+s_{*} B_{t}$ and $\operatorname{deg}\left(B_{r}\right)=18$. The formula of Hurwitz applied to $s$ and $t$ implies that either $\operatorname{deg}\left(B_{s}\right)=\operatorname{deg}\left(s_{*} B_{t}\right)=6$ and $p=1$ or $\operatorname{deg}\left(B_{s}\right)=8, \operatorname{deg}\left(s_{*} B_{t}\right)=2$ and $p=2$.

Since $\sigma$ factors through $\mathbb{P}\left(\Omega_{\mathrm{P}_{\mathrm{C}}^{2} \mid \mathrm{C}}^{1}(3)\right)$, then $s$ factors through $\mathbb{P}\left(\mathcal{O}_{\ell}(1) \oplus \mathcal{O}_{\ell}(2)\right)$. In any case the Tschirnhausen module of $s, \mathcal{E}_{s}$, is dual to $\mathcal{O}_{\ell}(1+h) \oplus \mathcal{O}_{\ell}(2+h)$ for some $h \in \mathbb{Z}$. Since $B_{s} \in\left|\operatorname{det}\left(\mathcal{E}_{s}\right)^{-2}\right|$ we get that $p=1$ and $h=0$. In particular the dual of the Tschirnhausen module of $S$ is $\Omega_{\mathbf{P}_{\mathrm{C}}^{2} \mathbf{c}}^{1}(3)$. It follows from Proposition 1.2 that $S$ is ruled with invariant $e(S)=-1$ over an elliptic curve.

On the other hand if $\mathcal{L} \in \operatorname{Pic}(S)$ is the Tschirnhausen module of $\tau$ the $\mathcal{O}_{A} \cong \omega_{A \mid \mathrm{C}} \cong$ $\omega_{S \mid C} \otimes \mathcal{L}$, thus $\tau$ is induced by a smooth and irreducible element of $\left|\omega_{S \mid C}^{-2}\right|$.

Conversely let $A$ be bielliptic and let $\varrho: A \rightarrow \mathbb{P}_{\mathrm{C}}^{2}$ be the corresponding cover factorizing as $\pi \circ i$. The double cover $\tau$ factors through $A \hookrightarrow \mathbb{V}\left(\omega_{S \mid c}\right) \subseteq \mathbb{P}\left(\mathcal{O}_{s} \oplus \omega_{s \mid c}\right)$ followed by the projection onto $S$.

In order to simplify notations, we will set $\overline{\mathbb{P}}:=\mathbb{P}\left(\Omega_{\mathrm{P}_{\mathrm{C}}^{2} \mathrm{C}}^{1}(3)\right)$. Let $\mathcal{F}:=\mathcal{O}_{\overline{\mathrm{p}}} \oplus\left(\mathcal{O}_{\overline{\mathbf{P}}}(1) \otimes\right.$ $\left.p^{*} \mathcal{O}_{\mathbb{P}_{\mathrm{C}}^{2}}(-3)\right)$ and $q: \mathbb{F}:=\mathbb{P}(\mathcal{F}) \rightarrow \overline{\mathbb{P}}$ be the projection. Since $\omega_{S \mid \mathrm{C}} \cong\left(\mathcal{O}_{\overline{\mathrm{P}}}(1) \otimes p^{*} \mathcal{O}_{\mathbb{P}_{\mathrm{C}}^{2}}(-3)\right)_{\mid S}$ then $\mathbb{P}\left(\mathcal{O}_{S} \oplus \omega_{S \mid \mathrm{C}}\right) \cong \mathbb{F} \times_{\overline{\mathrm{p}}} S$. Define $\mathcal{M}:=\mathcal{O}_{\mathbf{r}}(1) \otimes q^{*} p^{*} \mathcal{O}_{\mathbb{P}_{\mathbf{C}}^{2}}(3)$. The general morphism $q^{*} p^{*} \mathcal{E} \rightarrow \mathcal{M}$ is surjective, thus we get $f: \mathbb{F} \rightarrow \mathbb{P}$ inducing 


$$
\begin{aligned}
& u: U \cong \mathbb{P}\left(\mathcal{O}_{\overline{\mathbf{P}}}\right) \subseteq \mathbb{F} \rightarrow \mathbb{P}, \\
& f^{\prime}: \mathbb{P}\left(\mathcal{O}_{\overline{\mathbf{P}}}(1) \otimes p^{*} \mathcal{O}_{\mathbf{P}_{\mathbf{C}}^{2}}(-3)\right) \subseteq \mathbb{F} \rightarrow \mathbb{P}
\end{aligned}
$$

Fix $x \in \mathbb{P}_{\mathbf{C}}^{2}$. Then $u$ embeds linearly $U_{x}:=p^{-1}(x) \cong \mathbb{P}_{k(x)}^{1} \subseteq \mathbb{P}_{x}:=\pi^{-1}(x) \cong \mathbb{P}_{k(x)}^{4}$ and $f$ is the natural embedding $\mathbb{F}_{x}:=(p \circ q)^{-1}(x) \cong \mathbb{F}_{1} \subseteq \mathbb{P}_{x} \cong \mathbb{P}_{k(x)}^{4}$ as a cubic scroll. In particular $f$ is actually an embedding. By construction $A_{x}:=\varrho^{-1}(x)$ generates a subscheme $\Sigma_{x} \subseteq \mathbb{F}_{x}$ which is exactly the pull back of $S_{x}:=\sigma^{-1}(x)$ via $q_{\mid \mathbb{F}_{x}}: \mathbb{F}_{x} \rightarrow U_{x}$.

Each subscheme $A^{\prime} \subseteq A_{x}$ of degree at least 5 generates $\Sigma_{x}$. On the other hand each hyperplane $H \subseteq \mathbb{P}_{x}$ intersect all the fibres of $\mathbb{F}_{x}$ and $H \cap \mathbb{F}_{x}$ is a cubic curve, thus $\Sigma_{x} \nsubseteq H$. It follows that $A^{\prime} \nsubseteq H$. Hence $A_{x} \subseteq \mathbb{P}_{x}$ is an arithmetically Gorenstein subscheme (see [19, Lemma 4.2]).

We then obtain that the induced embedding $i: A \hookrightarrow \mathbb{P}$ coincides with the embedding given by the canonical factorization of $\varrho$ in the sense of Theorem 2.1 of [9].

In this case $q$ is induced by the projection of $\mathbb{P}$ onto $U$ from the subbundle $V$ generated by $\operatorname{im} f^{\prime}$. Necessarily there exists a locally free $\mathcal{O}_{\mathbf{P}_{\mathbf{C}}^{2}}$-sheaf $\mathcal{G}$ of rank 3 such that $V \cong \mathbb{P}(\mathcal{G})$.

Since $U$ and $V$ generate fibrewise $\mathbb{P}$ and $U \cap V=\emptyset$, then $\mathcal{E} \cong \mathcal{G} \oplus \Omega_{\mathrm{P}_{\mathrm{C}}^{2} \mathrm{C}}^{1}$ (3). Notice that such an isomorphism gives rise to a factorization

$$
\mathcal{O}_{\mathrm{P}_{\mathrm{C}}^{2}}(3) \mapsto \mathcal{G} \oplus \Omega_{\mathrm{P}_{\mathrm{C}}^{2} \mathrm{C}}^{1}(3) \rightarrow \mathcal{O}_{\mathrm{P}_{\mathrm{C}}^{2}}(3)
$$

of the identity on $\mathcal{O}_{P_{C}^{2}}$. Since $h^{0}\left(\mathbb{P}_{C}^{2}, \Omega_{P_{C}^{2} \mid C}^{1}\right)=0$, one can split the above sequence through $\mathcal{G}$, hence $\mathcal{G} \cong \mathcal{O}_{\mathrm{P}_{\mathrm{C}}^{2}}(3) \oplus \mathcal{H}$. As in the proof of Proposition 1.1 one easily checks that $\mathcal{H} \cong \Omega_{\mathrm{P}_{\mathrm{C}}^{2} \mid \mathrm{C}}^{1}(3)$.

Thus we have proved the following converse of Proposition 4.1.

Proposition 4.2. If $A$ is bielliptic, then there are subbundles $\mathbb{P}\left(\Omega_{\mathrm{P}_{\mathrm{C}}^{2} \mid \mathrm{C}}^{1}(3)\right) \cong U \subseteq \mathbb{P}$ and $\mathbb{P}\left(\mathcal{O}_{\mathbf{P}_{\mathbf{C}}^{2}}(3) \oplus \Omega_{\mathbf{P}_{\mathbf{C}}^{2} \mid}^{1}(3)\right) \cong V \subseteq \mathbb{P}$ such that

(i) $A \cap V=U \cap V=\emptyset$;

(ii) let $\bar{\pi}: \mathbb{P} \rightarrow U$ be the projection from $V$, and identify $S$ with its image inside $U$ : then $S=\bar{\pi}(A), \tau=\bar{\pi}_{\mid A}$ and $\sigma=\bar{\pi}_{\mid S}$.

\section{REFERENCES}

1. W. BARTH, Abelian surfaces with $(1,2)$-polarization, in Algebraic Geometry, Sendai, 1985 (T. Oda, ed., vol. Advanced Studies in Pure Mathematics 10, 1987).

2. W. Barth, C. Peters and A. Van de Ven, Compact complex surfaces (Springer, 1984).

3. A. Beauville, Surfaces algebriques complexes, Astérisque 54 (1978).

4. Ch. Birkenhake and $H$. LANGe, A family of abelian surfaces and curves of genus four, Manuscripta Math. 85 (1994), 393-407.

5. Ch. Birkenhake, H. Lange and D. van Straten, Abelian surfaces of type $(1,4)$, Math. Ann. 285 (1989), 625-646. 
6. E. Brieskorn and H. KnÖRRER, Plane algebraic curves (Birkhäuser, 1986).

7. G. Casnati, Covers of algebraic varieties II. Covers of degree 5 and construction of surfaces, J. Algebraic Geom. 5 (1996), 461-477.

8. G. Casnati, Covers of algebraic varieties III. The discriminant of a cover of degree 4 and the trigonal construction, Trans. Amer. Math. Soc. 350 (1998), 1359-1378.

9. G. CASNATI and T. Ekedahl, Covers of algebraic varieties I. A general structure theorem, covers of degree 3, 4 and Enriques surfaces, J. Algebraic Geom. 5 (1996), 439-460.

10. F. J. Gallego and B. P. Purnaprajna, Normal presentation on elliptic ruled surfaces, J. Algebra 186 (1996), 597-625.

11. R. HARTSHORNe, Algebraic geometry (Springer, 1977).

12. K. Hulek, Abelian surfaces in products of projective spaces, in Algebraic Geometry, L'Aquila, 1988 (A. J. Sommese, A. Biancofiore and E. L. Livorni, eds., Lecture Notes in Math. 1417, 1990).

13. K. Hulek and S. H. Weintraub, Bielliptic abelian surfaces, Math. Ann. 283 (1989), 411-429.

14. H. Lange and Ch. Birkenhake, Complex abelian varieties (Springer, 1992).

15. R. Miranda, Triple covers in algebraic geometry, Amer. J. Math. 107 (1985), 11231158.

16. C. OKONEK, M. SCHNEIDER and H. SPINDLER, Vector bundles on complex projective spaces (Birkhäuser, 1980).

17. R. Pardini, Triple covers in positive characteristic, Ark. Mat. 27 (1989), 319-341.

18. S. Ramanan, Ample divisorson abelian surfaces, Proc. London Math. Soc. 51 (1985), 231-245.

19. F. O. SCHREYer, Syzygies of canonical curves and special linear series, Math. Ann. 275 (1986), 105-137.

20. F. Serrano, Elliptic surfaces with an ample divisor of genus two, Pacific J. Math. 152 (1992), 187-199.

21. H. Tokunaga, Triple coverings of algebraic surfaces according to the Cardano formula, J. Math. Kyoto Univ. 31 (1991), 359-375.

22. F. Tovena, Abelian surfaces with polarization of type $(1,4)$, in Abelian varieties, Egloffstein 1993 (W. Barth, K. Hulek and H. Lange, eds., Walter de Gruyter, 1995).

Dipartimento di Matematica Pura ed Applicata

UniVersità degli StUdi di Padova

VIA BELZONI 7

I-35131 PaDOVA

ITALY

E-mail address: casnati@math.unipd.it 